\title{
Rheology and dynamics of vesicle suspension in comparison with droplet emulsion
}

\author{
G. Danker, C. Verdier and C. Misbah \\ Laboratoire de Spectrométrie Physique, \\ Université Joseph Fourier Grenoble I and CNRS (UMR5588), \\ 140 avenue de la physique, BP87 - 38402 Saint-Martin d'Hères cedex, France.
}

\begin{abstract}
This paper deals with the study of dynamics and rheology of a dilute suspension of vesicles. The study is analytical and is based on the small deformation theory. Vesicles in the small deformation limit exhibit, under shear flow, rich dynamics in comparison to droplets (in the regime where the droplet maintains its integrity). For example, droplets only assume a fixed orientation with respect to the flow, while vesicles undergo three types of motions: (i) tank-treading ( $t$, where the vesicle assumes a fixed orientation with respect to the flow, while the membrane makes a tanktreading motion, (ii) tumbling $(t b)$, which occurs as a saddle-node bifurcation from the tank-treading motion for a certain critical viscosity ratio, (iii) vacillating-breathing $(v b)$, where the vesicle long axis undergoes oscillations around the shear direction whereas its shape executes a breathing-like motion. This mode is found to coexist with tumbling in the high shear rate limit (or high capillary number $C_{a} \equiv \dot{\gamma} \tau$, where $\dot{\gamma}$ is the shear rate and $\tau$ is the relaxation time towards equilibrium shape of the vesicle). After analyzing these modes and comparing dynamics to droplets, we study rheology. It is found that the constitutive law, written in the co-moving frame, is nonlinear even to leading order. This markedly contrasts with droplet emulsion where the equation is linear to leading order. We make a link between rheology and the above three dynamical states. It is found that the effective viscosity undergoes a cusp singularity at the tumbling bifurcation (which happens at small enough $C_{a}$ ), while the normal stress differences collapse in the tumbling and VB regimes. At high enough $C_{a}$ the $t b$ transition is preceded by the $v b$ mode. We also report on shear thinning and the behavior of the normal stress difference as a function of $\dot{\gamma}$.
\end{abstract}




\section{INTRODUCTION}

Vesicles are closed phospholipidic membranes suspended in an aqueous solution. The membrane is made of a bilayer (Fig. 1), very much like cytoplasmic membranes of real cells. Vesicles are believed to mimic the mechanical and some of the viscoelastic and dynamical behaviors of red blood cells. The membrane may be in the fluid state (as is the case in physiological conditions), in which we are interested here, or in the gel state (at low enough temperature). In the fluid state each phospholipidic molecule may diffuse like a molecule in a usual fluid state does, the main difference is that the phospholipidic motion is confined to two dimensions. Due to its fluid character, the membrane does not resist to shear forces. Owing to the cohesive force between the phospholipids, the membrane behaves as a two dimensional incompressible fluid. The only possible (soft) mode is the bending mode of the membrane. The enclosed fluid, usually water (or water and some additives, such as sugar, or polymers in order to act on the internal viscosity), is also incompressible. Typical sizes of vesicle diameter lie in the range $1-100 \mu \mathrm{m}$, while the thickness of the membrane is of the order of few nanometers, so that the membrane may be viewed as a two dimensional surface.

The first important study of vesicles at equilibrium is due to Helfrich[1] who introduced the notion of curvature free energy of membranes. He introduced a model in which the cost in bending energy is given by

$$
F=\frac{\kappa}{2} \int H^{2} d A+\frac{\kappa_{g}}{2} \int K d A
$$

$H$ is the mean curvature, $H=1 / R_{1}+1 / R_{2}$, where $R_{1,2}$ are the principal radii of curvature, and $K$ is the Gauss curvature, equal to $1 /\left(R_{1} R_{2}\right)$. The integrals are performed along the membrane area $A$, and $\kappa$ and $\kappa_{g}$ have the dimension of an energy (as does $F$ ), and represent the bending rigidity and the Gaussian rigidity, respectively. Expression (1) may be inferred from some general invariance considerations: since energy is a physical quantity, it can not depend on reparametrization of the surface. The bending of the membrane may be represented by a $2 \times 2$ matrix (for example, $\nabla_{\mathbf{s}} \mathbf{n}$, where $\mathbf{n}$ is the unit vector normal to the membrane, and $\nabla_{\mathbf{s}}$ is the gradient along the membrane) that measures the strength of local bending. In two dimensions there are two invariants, which are the trace and the determinant of $\nabla_{\mathbf{s}} \mathbf{n}$. The trace is nothing but the mean curvature, and the determinant is the Gauss curvature. The energy should depend on these two quantities only. Furthermore, 


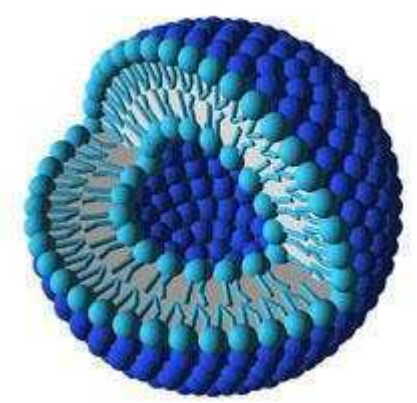

Figure 1: Schematic view of a vesicle made of a bi-layer of phospholipid molecules

if we view the membrane as a two dimensional sheet, both concave and convex shapes having the same amplitude of their mean curvature should have the same energy (to leading order where the internal structure of the membrane is ignored). Thus, the first plausible candidate in the free energy is $H^{2}$ [41]. It is known that the second term in (1) is a topological invariant by virtue of a theorem of differential geometry (Gauss-Bonnet theorem). That is to say, if one is not interested in a change of topology (like creation of two vesicles out of one, or transition from a closed membrane to a perforated membrane), then this contribution is a constant, and can be ignored. This is adopted here, since we do not want to account for topological changes.

Equilibrium shapes of vesicles then correspond to a minimum of $\kappa / 2 \int H^{2} d A$ subject to two constraints (i) fixed area (incompressible membrane), (ii) fixed volume (incompressible enclosed fluid). Mathematically this is dealt with by minimizing

$$
\frac{\kappa}{2} \int H^{2} d A+\zeta A+p V
$$

where $\zeta$ and $p$ are Lagrange multipliers enforcing constant area $A$ and constant volume $V$, respectively. Because the curvature energy is scale invariant changing lengths $\mathbf{r}$ by $\mu \mathbf{r}$ (where $\mu$ is a real constant) leaves the curvature energy invariant. Thus the only free parameter is the reduced volume $\nu=[V /(4 \pi / 3)] /[A / 4 \pi]^{3 / 2}$. This is a dimensionless number which is equal to one for a sphere, and $\nu<1$ for any other shape. As $\nu$ decreases from one, the Helfrich model reveals a variety of equilibrium shapes, as shown on Fig.2. For human red blood cells, for example, $\nu \sim 0.64$, and the equilibrium shape obtained from the Helfrich model is the discocyte one shown on Fig.2. The shapes shown on Fig.2 have been also observed experimentally. The Helfrich model has given rise to numerous studies both 


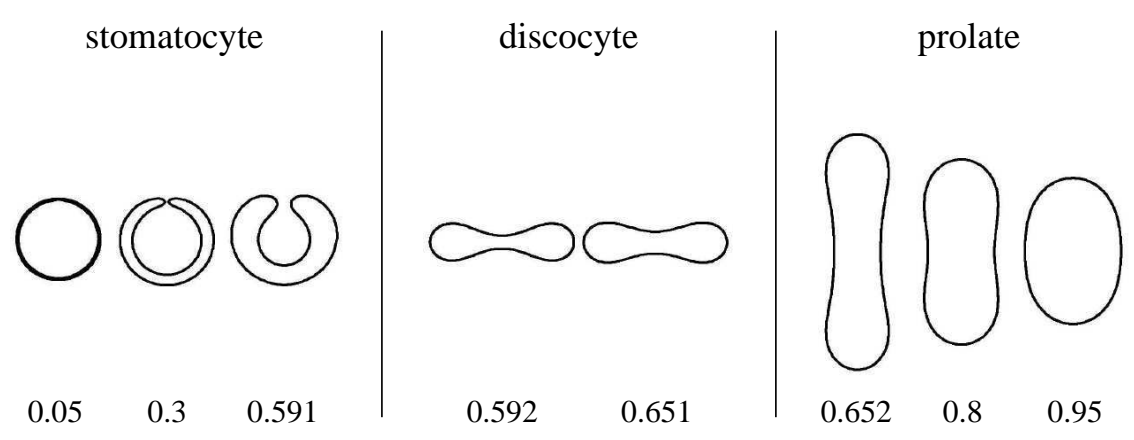

Figure 2: Equilibrium shapes for different reduced volume. Redrawn from [3].

theoretically and experimentally. The whole phase diagram[42] of equilibrium shapes is now fairly understood[2].

In most situations of interest, like in the context of transport of blood elements, nonequilibrium dissipative dynamics prevail. Studies on the nonequilibrium features of vesicles have began since about a decade. Since then they have induced a considerable amount of research both experimentally and theoretically[4-9]. The small deformation analytical theory, known for droplets, has been adopted for vesicles $[9,13]$. A brief account has been given recently regarding three types of dynamics: tanktreading $(t t)$, tumbling $(t b)$ and vacillating-breathing $(v b)[9]$. This theory serves as a basis for the derivation of the rheological law presented here. The first aim of the present paper is to provide an extended analysis of vesicle dynamics under shear flow and to compare the outcome with those of droplet dynamics. We shall then focus on the rheology of vesicles and discuss the main difference with droplets emulsion rheology. We shall also make a link between rheology, the microscopic dynamics and bifurcations exhibited by vesicles. A natural extension of the present theory will be discussed.

\section{VESICLES IN AN EXTERNAL FLOW}

We shall now discuss the behavior of a vesicle in an external flow field. We start by identifying the important physical ingredients of the problem, then show how relevant results can be obtained for quasispherical vesicles using asymptotic expansion techniques, and finally discuss the dynamics of vesicles in shear flow as observed in recent experiments and described by the theoretical approaches. 


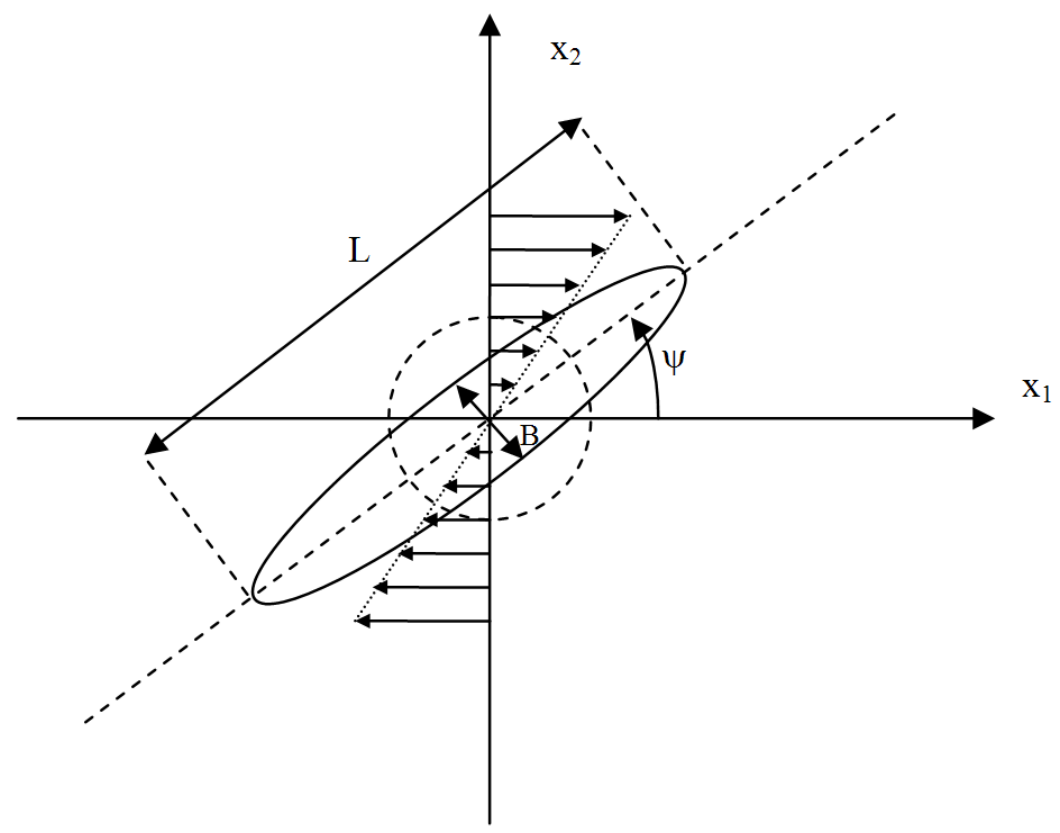

Figure 3: Sketch of the deformation of a vesicle or droplet in a shear flow $\mathbf{v}=\dot{\gamma} x_{2} \mathbf{e}_{1}$.

\section{A. The physical model}

Vesicles are characterized by two important quantities, both of which can be regarded as constant under usual experimental conditions: (1) The volume $V$ and (2) the dimensionless excess area $\Delta=4 \pi\left(A-A_{0}\right) / A_{0}$, where $A$ is the surface area of the vesicle and $A_{0}$ is the area of a sphere having the same volume. $\Delta$ is related to the reduced volume via $\Delta=4 \pi\left[\nu^{-2 / 3}-1\right]$. For $\Delta=0(\nu=1)$ the vesicle is spherical, cannot be deformed and acts like a solid body in a stationary flow. For $\Delta>0$ the equilibrium shape is not a sphere and the dynamics in an external flow is nontrivial.

In common experiments, the Reynolds number of the flow is much smaller than 1 . The dynamics of the flow is thus adequately described by the Stokes equations,

$$
\eta_{\alpha} \nabla^{2} \mathbf{u}=\nabla p
$$

where $\eta_{0}$ is the viscosity of the suspending fluid and $\eta_{1}$ is the viscosity of the fluid inside. We define as usually the ratio $\lambda \equiv \eta_{1} / \eta_{0}$. We can assume that the fluids are incompressible, thus

$$
\nabla \cdot \mathbf{u}=0
$$


The membrane of the vesicle is subject to bending forces (due to its bending rigidity $\kappa$ ) and to normal and tangential stresses, which enforce local membrane incompressibility. The force density at the membrane is obtained as a functional derivative of (2) with respect to the vector position $\mathbf{r}$ of the membrane. In addition, $\zeta$ which is constant at equilibrium, becomes now a space and time dependent multiplier. Indeed, under flow, the hydrodynamical forces vary, generally, from one point to another (since the flow field is inhomogeneous). These forces tend to dilate or compress the membrane. Since cohesive forces are such that each local area must be incompressible, one must adapt locally the Lagrange multiplier. In addition, since the flow may vary from one time to the other, we must impose a time-dependent Lagrange multiplier as well. Finally, since under flow we impose (4) there is no need to enforce the enclosed volume by a Lagrange multiplier (now the pressure $p$ may be viewed as the quantity that adapts itself to fulfil incompressibility). Having this in mind, the functional derivative can be performed and yields the force [13]

$$
\mathbf{f}=\left\{\kappa\left[2 H\left(2 H^{2}-2 K\right)+2 \Delta_{\mathrm{B}} H\right]-2 \zeta H\right\} \mathbf{n}+g^{i j} \mathbf{R}_{i} \partial_{j} \zeta
$$

$g^{i j}$ is the inverse of the metric, and $\Delta_{\mathrm{B}}$ is the Laplace-Beltrami operator. Finally, $\mathbf{n}$ is the normal to the membrane and $\mathbf{R}_{1}, \mathbf{R}_{2}$ are tangent vectors.

At the vesicle surface, the membrane forces must balance the fluid stresses

$$
\sigma_{i j}=-p \delta_{i j}+\eta_{\alpha}\left(\partial_{i} u_{j}+\partial_{j} u_{i}\right), \quad \alpha=0,1
$$

and the fluid velocities must be continuous and coincide with the membrane velocity (an impermeable membrane is assumed).

\section{B. Small deformation theory}

Analytical progress is possible for the case of almost spherical vesicles, where the excess area $\Delta$ is small. We describe the surface of the vesicle by

$$
\mathbf{r}=r_{0}(1+\epsilon f) \mathbf{e}_{r}
$$

where the function $f(\theta, \phi)$ measures the departure from the spherical shape and $\mathbf{e}_{r} \equiv \mathbf{r} /|\mathbf{r}|$ is the radial unit vector. The prefactor $\epsilon$ is a small quantity and can be related to the excess area via $\epsilon=\Delta^{1 / 2}$. 
We decompose the function $f$ in terms of surface spherical harmonics of order 2 , which is the leading contribution in the expansion of the surface perturbation:

$$
f(\theta, \phi)=F_{i j} \frac{\partial^{2} r^{-1}}{\partial x_{i} \partial x_{j}} r^{3}, \quad r=\sqrt{x_{1}^{2}+x_{2}^{2}+x_{3}^{2}} .
$$

The amplitudes $F_{i j}(t)$ describe the shape dynamics of the vesicle and are obtained from the self-consistent solution outlined below. The zeroth and first order modes, which describe dilatation and translation, respectively, do not contribute to leading order (in the co-moving frame).

We assume that the vesicle is subject to a shear flow of the form $\mathbf{v}_{0}=\dot{\gamma} x_{2} \mathbf{e}_{1}$ (see Fig. 3). The total velocity field outside the vesicle can then be written as $\mathbf{v}=\mathbf{v}_{0}+\mathbf{u}$, where $\mathbf{u}$ is the perturbation of the field due to the presence of the vesicle. Likewise, we write for the velocity field within the vesicle: $\overline{\mathbf{v}}=\boldsymbol{\omega} \times \mathbf{r} / 2+\overline{\mathbf{u}}$, where $\boldsymbol{\omega}$ is the vorticity. Following Lamb [14], we write an ansatz for the unknown perturbation of the velocity field outside the vesicle in the form

$$
\mathbf{u}=\sum_{n=0}^{\infty} \nabla \chi_{-n-1} \times \mathbf{r}+\nabla \phi_{-n-1}-\frac{n-2}{2 n(2 n-1)} r^{2} \nabla p_{-n-1}+\frac{n+1}{n(2 n-1)} \mathbf{r} p_{-n-1}
$$

and inside the vesicle

$$
\overline{\mathbf{u}}=\sum_{n=0}^{\infty} \nabla \bar{\chi}_{n} \times \mathbf{r}+\nabla \bar{\phi}_{n}+\frac{n+3}{2(n+1)(2 n+3)} r^{2} \nabla \bar{p}_{n}-\frac{n}{(n+1)(2 n+3)} \mathbf{r} \bar{p}_{n} .
$$

The functions $\bar{p}_{n}, \bar{\phi}_{n}$, and $\bar{\chi}_{n}$ in the Lamb solution are solid spherical harmonics of order $n$ and $p_{-n-1}, \phi_{-n-1}, \chi_{-n-1}$ are solid spherical harmonics of order $-n-1$ [15]. Splitting off their $r$-dependence, we write $\chi_{-n-1}=r^{-n-1} Q_{n}, \phi_{-n-1}=r^{-n-1} S_{n}$, and $p_{-n-1}=r^{-n-1} T_{n}$. Likewise for the quantities within the vesicle: $\bar{\chi}_{n}=r^{n} \bar{Q}_{n}, \bar{\phi}_{n}=r^{n} \bar{S}_{n}$, and $\bar{p}_{n}=r^{n} \bar{T}_{n}$. The precise values of the functions $Q_{n}, S_{n}, \ldots$ (which are surface spherical harmonics and thus depend only on the angles) are determined by the boundary conditions at $r=r_{0}$. Note that to leading order only the modes related to $n=2$ enter the calculation [9].

Like the function $f$ in Eq. (8), the functions $Q_{2}, \ldots$ are expressed in terms of rank-two tensors:

$$
Q_{2}=Q_{i j} \frac{\partial^{2} r^{-1}}{\partial x_{i} \partial x_{j}} r^{3}, \ldots
$$

The Lagrange multiplier $\zeta$ is written as

$$
\zeta=Z_{0}+Z_{i j} \frac{\partial^{2} r^{-1}}{\partial x_{i} \partial x_{j}} r^{3} .
$$


Now we apply the boundary conditions to find the unknown velocity field. Continuity of the velocity across the membrane gives rise to the equations

$$
\begin{aligned}
\bar{T}_{i j}-T_{i j}+10 \bar{S}_{i j} & =\frac{5}{3} e_{i j}, \\
\frac{2}{7} \bar{T}_{i j}-T_{i j}+4 \bar{S}_{i j}+6 S_{i j} & =\frac{2}{3} e_{i j},
\end{aligned}
$$

where $e_{i j}$ is the symmetric part of the velocity gradient of the imposed flow.

Similarly, the stress balance at the membrane yields

$$
\begin{aligned}
\lambda \bar{T}_{i j}+\frac{3}{2} T_{i j}+10 \lambda \bar{S}_{i j} & =\frac{5}{3} e_{i j}+Z_{i j}-4\left(Z_{0}+6 \kappa\right) F_{i j}, \\
3 T_{i j}-\frac{1}{7} \lambda \bar{T}_{i j}+4 \lambda \bar{S}_{i j}-24 S_{i j} & =\frac{2}{3} e_{i j}-2 Z_{i j}-4\left(Z_{0}+6 \kappa\right) F_{i j} .
\end{aligned}
$$

Eqs. $(13,14,15,16)$ permit the determination of $T_{i j}, S_{i j}, \bar{T}_{i j}, \bar{S}_{i j}$ as a function of $F_{i j}$ and $Z_{i j}$.

Local membrane incompressibility provides an additional relation,

$$
\frac{3}{7} \bar{T}_{i j}+2 \bar{S}_{i j}=0
$$

which fixes $Z_{i j}$.

$>$ From the condition that at $r=r_{0}$ the fluid velocities must coincide with the membrane velocity we obtain the following nonlinear evolution equation for the amplitudes $F_{i j}(t)$ :

$$
\epsilon \frac{\mathcal{D} F_{i j}}{\mathcal{D} t}=\frac{20 e_{i j}}{23 \lambda+32}-\frac{24\left(Z_{0}+6 \kappa\right)}{23 \lambda+32} F_{i j}
$$

where $\mathcal{D} / \mathcal{D} t$ is the Jaumann derivative

$$
\frac{\mathcal{D} \mathbf{M}}{\mathcal{D} t}=\frac{D \mathbf{M}}{D t}+\frac{1}{2}[\omega \mathbf{M}-\mathbf{M} \omega]
$$

Here $\mathbf{M}$ is any second order tensor, $D / D t$ is the usual material derivative, and $\omega=(\nabla \mathbf{v}-$ $\left.\nabla \mathbf{v}^{T}\right) / 2$ is the vorticity tensor.

Eq. (18) still contains the isotropic part $Z_{0}(t)$ of the membrane tension as a parameter. Its value is determined by the demand that the dynamics of the shape perturbation must conform to the available excess area $\Delta$. From this condition it follows that $Z_{0}+6 \kappa=$ $8 \pi \dot{\gamma} F_{12} / \Delta$. The equation for the dynamics of the vesicle shape thus becomes

$$
\epsilon \frac{\mathcal{D} F_{i j}}{\mathcal{D} t}=\frac{20 e_{i j}}{23 \lambda+32}-\frac{192 \pi \dot{\gamma}}{23 \lambda+32} \frac{F_{12} F_{i j}}{\Delta} .
$$

Note that this evolution equation is nonlinear even at leading order due to the coupling of the modes from the area constraint. This is in marked contrast with the corresponding 
equations for droplets and elastic capsules, which, to leading order, are linear (see section devoted to droplets).

The solutions of the evolution equation (20), which describe the behavior of a vesicle in a shear flow, will be discussed in the next section.

\section{Dynamics under shear flow}

Recently several experimental studies have focused on vesicle dynamics in a shear flow [16-19]. Some experiments have been carried out for $\lambda=1$, which is an important special case. Here the vesicle assumes a time-independent ellipsoidal shape and orients under a well-defined angle $\psi_{0}$ with respect to the flow direction, while the membrane, because it is fluid, undergoes a tank-treading like motion (Fig. 4a). The small deformation theory [13] makes a prediction for the angle $\psi_{0}$, which agrees well with experimental measurements [19].

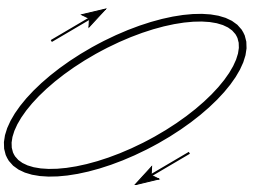

a)

b)
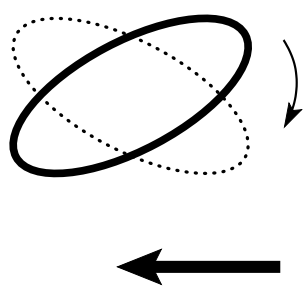

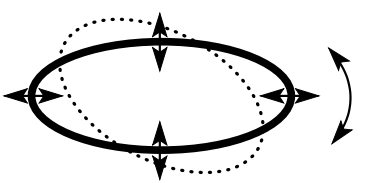

c)

Figure 4: Schematic representation of tank-treading (a), tumbling (b) and vacillating-breathing (c) of a vesicle in a shear flow.

Here, however, we want to concentrate on cases $\lambda>1$, which give rise to richer dynamics. For simplicity we concentrate on the dynamics in the plane of the shear, which is characterized by only three components, $F_{11}, F_{12}$, and $F_{22}$. These components are still coupled by the area constrained, so that the dynamics of the vesicle can be expressed in terms of only two independent quantities. Following previous works $[9,10]$, we describe the dynamics in terms of the vesicle orientation angle $\psi$ and its deformation $R$. The two quantities are defined by

$$
\begin{aligned}
& R \cos 2 \psi=\sqrt{\frac{6 \pi}{5}}\left(F_{11}-F_{22}\right), \\
& R \sin 2 \psi=2 \sqrt{\frac{6 \pi}{5}} F_{12} .
\end{aligned}
$$


$>$ From Eq. (20) we can now extract two coupled equations that describe the dynamics in terms of these two variables:

$$
\begin{aligned}
& \epsilon \partial_{t} R=h\left[1-4 \frac{R^{2}}{\Delta}\right] \sin 2 \psi, \\
& \epsilon \partial_{t} \psi=-\frac{1}{2}+\frac{h}{2 R} \cos 2 \psi
\end{aligned}
$$

with $h=60 \sqrt{2 \pi / 15} /(32+23 \lambda)$.

These equations offer a tank-treading solution (which is characterized by fixed deformation and orientation) with

$$
\begin{aligned}
& R_{0}=\frac{\sqrt{\Delta}}{2} \\
& \psi_{0}= \pm \frac{1}{2} \cos ^{-1}\left[\frac{23 \lambda+32}{120} \sqrt{\frac{15 \Delta}{2 \pi}}\right] .
\end{aligned}
$$

The solution for $\psi_{0}$ has two branches, where the "+" solution is stable and the "-" solution is unstable [9]. The vesicle deformation $D$ is defined by $D=\frac{L-B}{L+B}$, where $L$ and $B$ are respectively the major and the minor axes of vesicle. We find that

$$
D=\sqrt{\frac{15 \Delta}{32 \pi}} .
$$

This is independent of the viscosity ratio.

If one concentrates only on the stable branch, one can also write from Eq.(26)

$$
\psi_{0}=\frac{\pi}{4}-\frac{1}{2} \tan ^{-1}\left[\frac{4 h^{2}-\Delta}{\Delta}\right]^{-1 / 2} .
$$

This writing will be useful for comparison with the droplet problem. Note that for $\Delta=0$ (sphere) the orientation angle is $\pi / 4$ (the orientation of maximum straining), otherwise the orientation angle is always smaller than $\pi / 4$.

There is a significant difference with droplets. For example, if $\Delta=0$, a vesicle can not deform, due to its membrane inextensibility. For an initially spherical drop, the deformation is possible due to migration of molecules from the bulk towards the surface. The underlying bulk plays the role of reservoir for the surface. The higher the shear rate, the larger is the deformation, as we shall see later. Since there is no reservoir of phospholipid, for a vesicle to deform, one needs necessarily $\Delta \neq 0$, and the maximum deformation that is attained is fixed by the excess area from a sphere, as given by Eq.(27). 
As the viscosity ratio $\lambda$ approaches the critical value (defined by $4 h^{2}=\Delta$ )

$$
\lambda_{c}=-\frac{32}{23}+\frac{120}{23} \sqrt{\frac{2 \pi}{15 \Delta}}
$$

the two solutions given by (26) (or that given by (28) and the opposite branch) vanish via a saddle-node bifurcation (see Fig. 5). Above $\lambda_{c}$ there is hence no fixed orientation solution (usually called tank-treading solution), instead an unsteady tumbling motion is found.

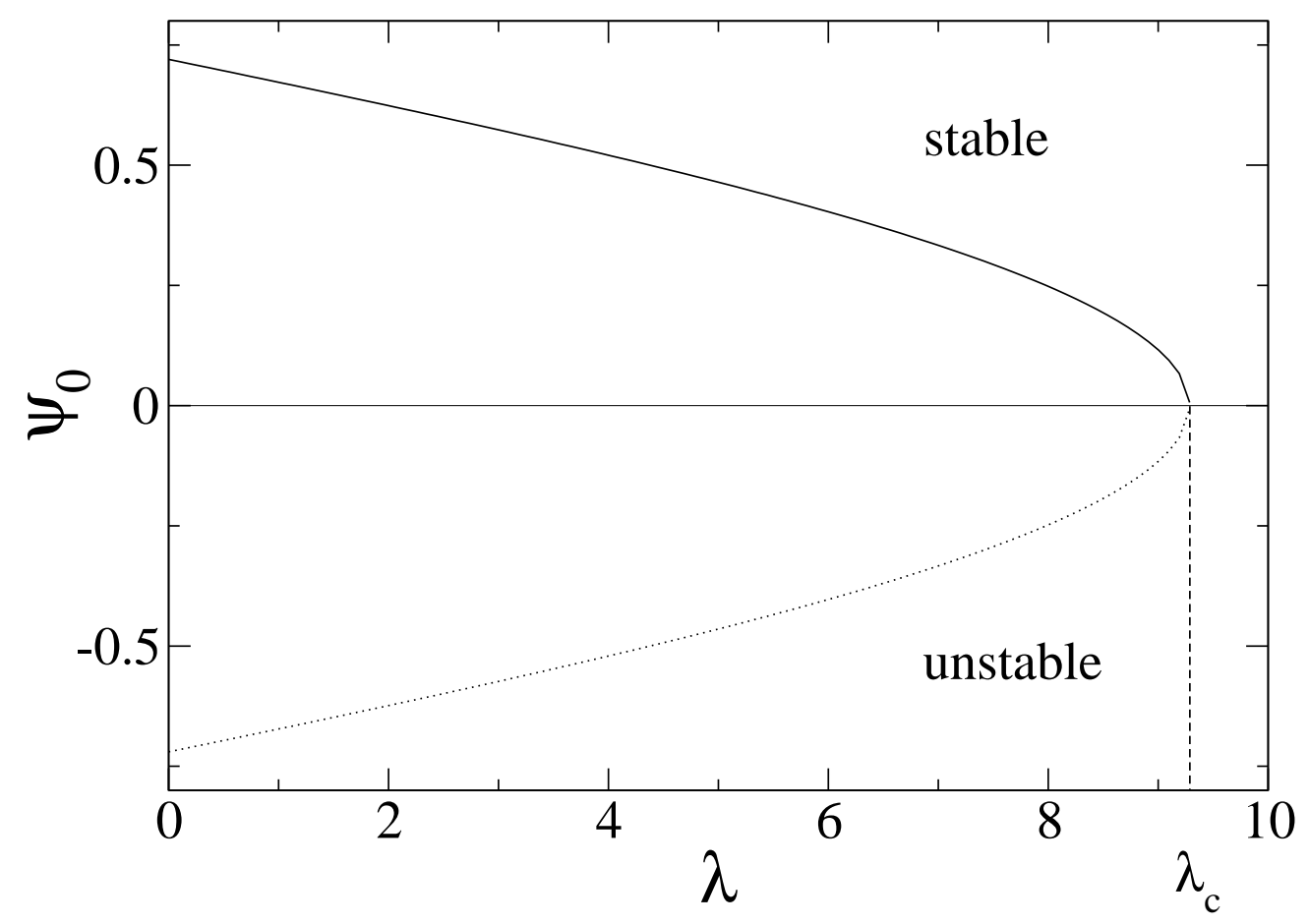

Figure 5: The stationary orientation angle $\psi_{0}$ of the tank-treading vesicle as a function of the viscosity ratio $\lambda$ for $\Delta=0.1$. The solid line represents the stable solution and the dotted line the unstable one. At the critical value $\lambda_{\mathrm{c}}$ both solutions vanish via a saddle-node bifurcation.

In fact, two types of dynamics can be distinguished for $\lambda>\lambda_{\mathrm{c}}$. The better-known motion is $t b$. Here the long axis of the vesicle rotates in the direction of the rotational component of the applied shear, while the shape undergoes slight periodic changes (see Fig. 4b for an illustration and Fig. 6 for the dynamics of the orientation angle). This type of motion has been studied in several experiments [18, 19] and comparisons with the Keller and Skalak [20] theory (which assumes undeformable ellipsoidal particles) have been attempted. It turns out that the Keller and Skalak theory is reasonably good for small enough shear rates, whereas at higher shear rates, deformation becomes important [18]. 


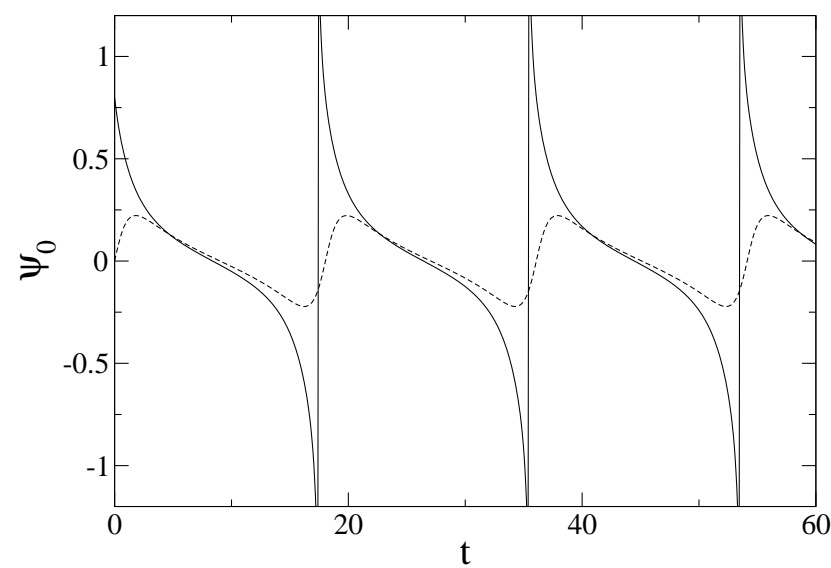

Figure 6: The dynamics of the orientation angle $\psi_{0}$ for tumbling (solid line) and vacillatingbreathing (dashed line). Parameters are: $\Delta=0.1, \lambda=10$. The curves are distinct because the initial conditions lie in different basins of attraction.

The other type of dynamics has been found only recently (theoretically[9] and experimentally[19]). It has been called vacillating-breathing $(v b)[9]$. This state corresponds to a situation where the long vesicle axis oscillates about the flow direction, while the shape performs a breathing motion (see Fig. 4 for an illustration and Fig. 6 for the dynamics of the orientation angle). According to the leading-order theory presented here, this motion coexists with $t b$, having its own basin of attraction. Very recently several theoretical studies have elucidated this point further[10-12]. In [12] it is explained how a consistent higher order theory must be performed. Here we shall report on some new features not yet reported on (See Fig. 12 and Fig. 13).

In the refined theories (which take higher-order contributions into account), the coexistence of $t b$ and $v b$ is expected for high capillary number $\left(C_{a} \equiv \dot{\gamma} \tau\right.$, where $\tau$ is the relaxation, time towards equilibrium). For very small $C_{a}$ there is a direct transition from tank-treading motion to $t b$ whereas for intermediate values there exists a first critical viscosity ratio $\lambda_{c}^{1}$ where tank-treading ceases to exist and $v b$ (or $t b$ ) starts and a second critical ratio $\lambda_{c}^{2}$ where $v b$ stops and $t b$ begins (see Fig. 7). This type of behavior (that is the $v b$ mode takes place beyond a certain critical $C_{a}$ ) agrees with the experiments[19]. Furthermore in Mader et al.[18] a mode referred too as "transition motion" bearing strong similarities with the $v b$ mode was reported. The transition motion occurs close to the $t b$ regime. It was also stated in [19] that the $v b$ mode takes place in the close vicinity of the $t b$. The higher order theory shows (see Fig.7) that (for $\Delta=0.5$ ) the $v b$ mode appears at $\lambda \sim 5.3$ at high enough $C_{a}$ and the $t b$ at 
$\lambda \simeq 6.5$. Furthermore, we have found that the higher order theory[12] shows that at about $C_{a} \simeq 100$ there is a coexistence between the $v b$ mode and the $t b$ one (transition towards one or the other depends on initial conditions), as presented in the original theory[9]. Note that these values of $C_{a}$ (and even much larger values) are quite accessible in experiments (see Mader et al.[18]). While the $t b$ mode has been extensively studied experimentally[18], a systematic experimental analysis of the $v b$ mode is lacking.

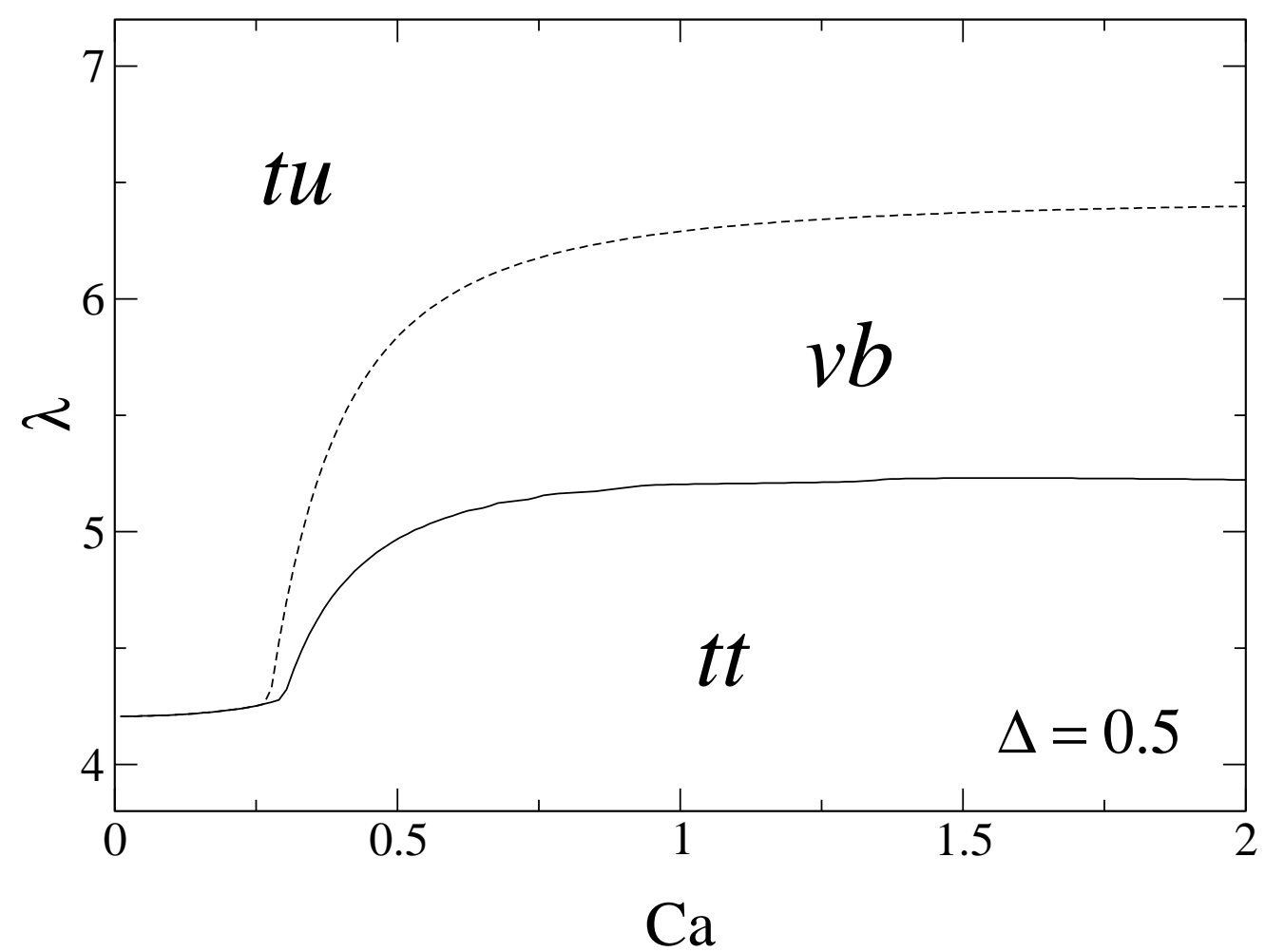

Figure 7: The three different domains of vesicle dynamics as a function of the capillary number $C a$ and the viscosity ratio $\lambda$ : tank-treading $(t t)$, tumbling $(t u)$, and vacillating-breating $(v b)$. The solid line corresponds to $\lambda_{c}^{1}$, the dashed line to $\lambda_{c}^{2}$ (see text).

\section{DYNAMICS OF NEWTONIAN DROPLETS IN A SHEAR FLOW}

We would like to compare the dynamics of vesicles to that of a drop. The deformation of such a Newtonian droplet (viscosity $\eta_{1}$ ) immersed in another fluid (viscosity $\eta_{0}$ ) during shearing motion (Fig.3) depends on the competition between the shear forces and the capillary ones. The relevant dimensionless number is the so-called capillary number $C a^{d}=\frac{a \dot{\gamma} \eta_{0}}{\Gamma} \equiv=\dot{\gamma} \tau^{d}\left(\tau^{d}\right.$ is the drop relaxation time towards equilibrium), where $\Gamma$ is the 
interfacial tension and $a$ is the initial radius of the droplet, assumed to be initially spherical. The viscosity ratio $\lambda$ also affects the droplet dynamics.

Predictions of the orientation and deformation of fluid droplets have been considered in shear and hyperbolic flows at low Reynolds numbers and small deformations [15, 21-24]. When the shear rate is suddenly applied, the droplet undergoes damped shape oscillations $[15,25]$, until it takes a stable ellipsoidal shape and stable orientation [15, 23]. Therefore, the droplet vacillates by changing its main dimensions and rotates at the same time around some angular position. These oscillations are damped with a characteristic relaxation time $\tau=\frac{a \eta_{1}}{\Gamma}$.

To solve for the flow fields, one needs to find the velocity vector fields which are solutions (inner and outer) of the Stokes equations together with proper boundary conditions at the interface, i.e. continuity of velocities, continuity of tangential stresses, and jump of normal stresses balanced by interfacial forces (instead of bending forces for vesicles). Unlike vesicles, no constraint on the droplet area is to be imposed [43].

The general flow solution is given by the Lamb solution, as presented in the section devoted to vesicles. The physics of a drop and that of a vesicle (basically due to membrane local incompressibility) leads to different dynamics. For example, the flow within the droplet is found to exhibit recirculations. If a spherical drop is, for example, falling under gravity in a quiescent fluid, the flow field shows two vorticies. In contrast, a spherical vesicle would behave exactly as a rigid body, in that there should be no circulation inside. In a shear flow, for a spherical vesicle the enclosed fluid will execute a solid-like rotation, while this is not the case for a droplet (see Fig.8).

The analogue of Eq.(20) for a droplet is given by [26]

$$
\epsilon^{\prime} \frac{\mathcal{D} F_{i j}}{\mathcal{D} t}=\frac{5}{3(2 \lambda+3)} e_{i j}-\frac{40(\lambda+1)}{(2 \lambda+3)(19 \lambda+16)} F_{i j}
$$

where $\epsilon^{\prime}$ is a small parameter ensuring the small strength of the drop deformation about a sphere. It is shown in Ref.[26] that generally $\epsilon^{\prime} \sim C a^{d}$. This means that the small deformation theory is valid for a small enough capillary number. The first important difference between Eq.(30) and Eq.(20) is the fact that (20) is nonlinear in the deformation $F_{i j}$, while it is linear for a drop (Eq. (30)). This is traced back to the local area constraint for vesicles. It is precisely this nonlinearity that is the source of rich dynamics and bifurcations for vesicles. 

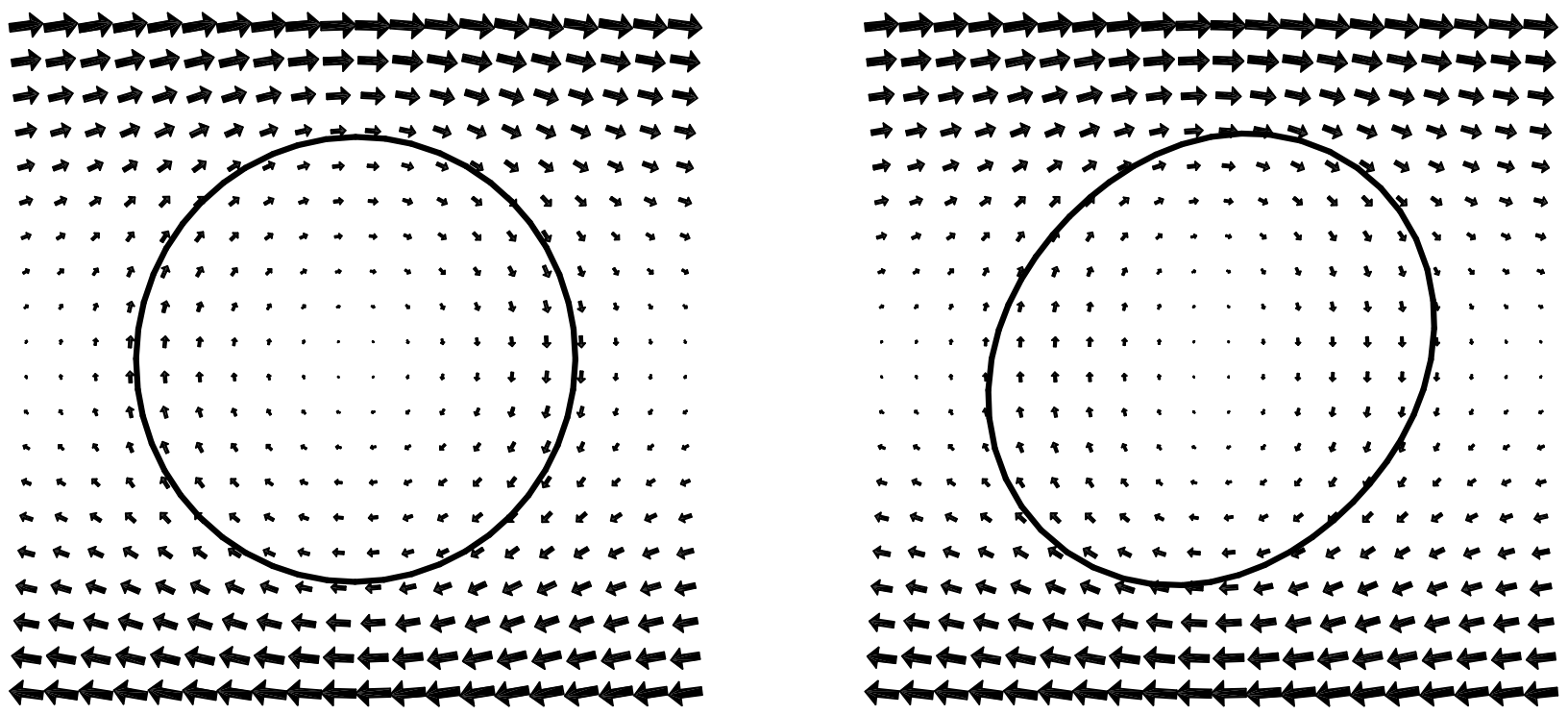

Figure 8: Left: Flow field for a vesicle with vanishing excess area. The vesicle behaves like a solid sphere. Right: Flow field for a droplet. The droplet deforms under the action of the shear flow.

The droplet deformation $D^{d}=(L-B) /(L+B)$ is given by

$$
D^{d}=\frac{5(19 \lambda+16)}{4(\lambda+1) \sqrt{(19 \lambda)^{2}+\left(\frac{20}{C a^{d}}\right)^{2}}}
$$

and the corresponding orientation angle $\psi_{0}^{d}$ at equilibrium, is given by

$$
\psi_{0}^{d}=\frac{\pi}{4}-\frac{1}{2} \tan ^{-1}\left(\frac{19 \lambda C a^{d}}{20}\right)
$$

This agrees with the previous developments of Taylor [22]. In particular, at very small deformations (small $C a^{d}$ ), one recovers the classical result,

- $\psi_{0}^{d}=\frac{\pi}{4}$

- $D^{d}=C a^{d} \frac{19 \lambda+16}{16 \lambda+16}$.

and for large viscosity ratios $\lambda$,

- $\psi_{0}^{d} \rightarrow 0$

- $D^{d}=\frac{5}{4 \lambda}$.

Recent work [27] has shown that the third axis $W$ in the $x_{1}-x_{3}$-plane is not necessarily conserved. The above authors propose a new phenomenological model for a second order 
tensor $\mathbf{S}$ whose eigenvalues represent the square semi-axes of the ellipsoidal drop. The evolution equation uses Jaumann derivatives, as well as "ad-hoc" functions. It can be used to predict transient evolutions and steady states. The two previous limits (slow flows at small $C a^{d}$, and high viscosity ratios $\left.\lambda\right)$ are also recovered. Analytic formulae for the principal axes $L, B$, and $W$ are found in terms of $C a^{d}$ and $\lambda$.

There are several specific differences with the vesicle problem. (i) Firstly, while for vesicles $D$ (Eq. (27)) is independent of $\lambda$, for droplets the deformation (Eq.(31)) critically depends on $\lambda$. For vesicles the deformation saturates due to the available excess area only, while for drops (and for fixed $C a^{d}$ ), the deformation tends to $D^{d} \sim 5 / 4 \lambda$. That is to say a large $\lambda$ ensures the saturation of the drop deformation (see below also). Indeed, in order for the drop to deform further, the liquid inside must flow to allow for molecules to migrate from the bulk towards the surface. This flow is too energetically costing for large $\lambda$. In particular (see below) beyond a certain critical $\lambda$, it becomes impossible to break the drop for any shear rate (or any capillary number). (ii) For droplets, for a given $C a^{d}$, when $\lambda$ is too large, the drop becomes stable and its orientation angle $\psi^{d} \rightarrow 0$, i.e. the drop aligns with the flow. The situation is completely different with vesicles, as shown in equation (28). For a fixed $\Delta$, and for $\lambda<\lambda_{c}$ (see Eq.(29)) the orientation angle (see also Fig.6) decreases from $\pi / 4$ down to zero when $\lambda=\lambda_{c}$. For $\Delta \sim 0.1-1$ (corresponding to $0.8-8 \%$ of relative excess area) where the small deformation theory is expected to make sense, $\lambda_{c} \sim 9-2$. This is in the range of values where a drop aligns with the flow. (iii) While both for drops and vesicles $\psi_{0} \rightarrow 0$ for a large enough $\lambda$, the way this occurs is completely different for both cases. From Eq.(32) the zero angle is strictly attained only for $\lambda \rightarrow \infty$ (see Fig.9). For vesicles the zero angle is attained for a finite $\lambda=\lambda_{c}$ (see Eq.(29)); see Fig.6. In addition it is clear that the topology of the curve is different. For vesicles, it shows a square root singularity at $\lambda_{c}$, whereas no such signature is exhibited for drops. The square root singularity is nothing but the fold catastrophe (or a saddle-node bifurcation) exhibited by vesicles. This corresponds to the $t b$ bifurcation. (iv) In addition to the existence of a $t t-t b$ bifurcation, the vesicle exhibits another type of of dynamics, namely the $v b$ mode discussed in the previous section.

The drop problem exhibits another feature, which is the breakup upon a finite shear rate. For vesicles breakup is possible, too, but the mechanism is quite different and the problem is still being explored [28]. For drops, while increasing the shear rate, the drop deforms further and further until it breaks. For drops of typical vesicle size $(\sim 100 \mu \mathrm{m})$ the 


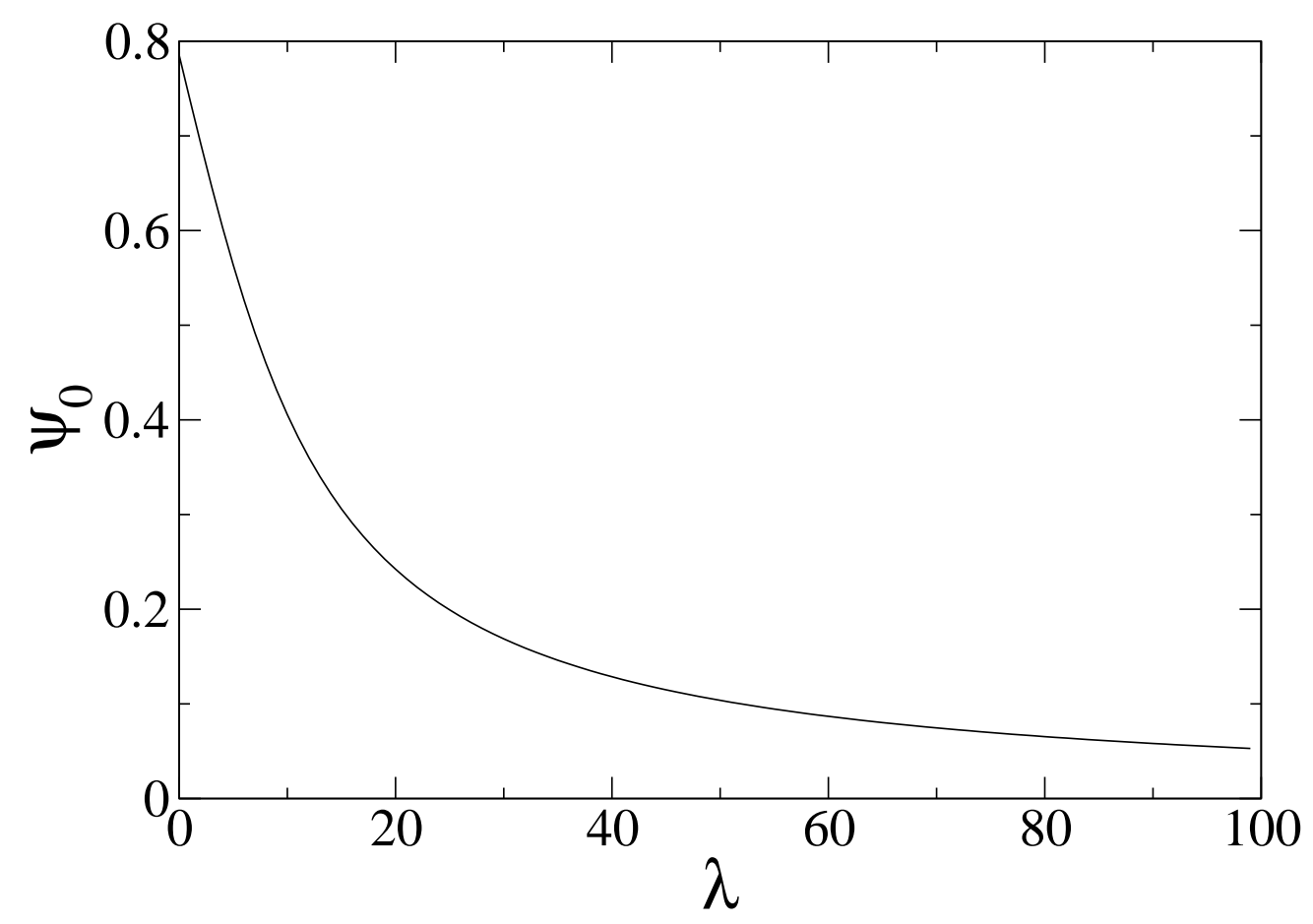

Figure 9: The orientation angle $\psi_{0}$ of a drop for a given $C a=0.1$

shear rate required for drop breakup is approximately $2000 \mathrm{~s}^{-1}$. The fluid inside the drop migrates toward the surface, and this phenomenon does not require, for example, acting on the molecular distance. For a vesicle to break, it is needed to apply forces close enough to cohesive forces at the membrane. This implies acting on the molecular cohesion. It is possible, in some circumstances, to create pores [29] on the membrane, which may then widen and cause breakup of the vesicle.

Let us briefly discuss large droplet deformations under shear flow, until droplet breakup is obtained. This corresponds to the well-known data obtained by Grace [30], in a large range of viscosity ratios $10^{-6} \leq \lambda \leq 10$. Critical values of the capillary number $C a_{c r}^{d}$ are shown in Fig.10. Clearly there is no breakup in shear when $\lambda \geq 3.5$ roughly. Also shown on this figure are typical droplets shapes for stable and unstable situations (leading to breakup). Grace also investigated many other parameters experimentally such as the critical drop deformation at burst, the breakup time, the number of fragments as well as their size.

On the theoretical side, the onset of bursting has been investigated by Barthès-Biesel and Acrivos [31] using a perturbation method up to the second power $\left(C a^{d}\right)^{2}$, followed by a linear stability analysis to determine the onset of bursting. This analysis is in good agreement with 


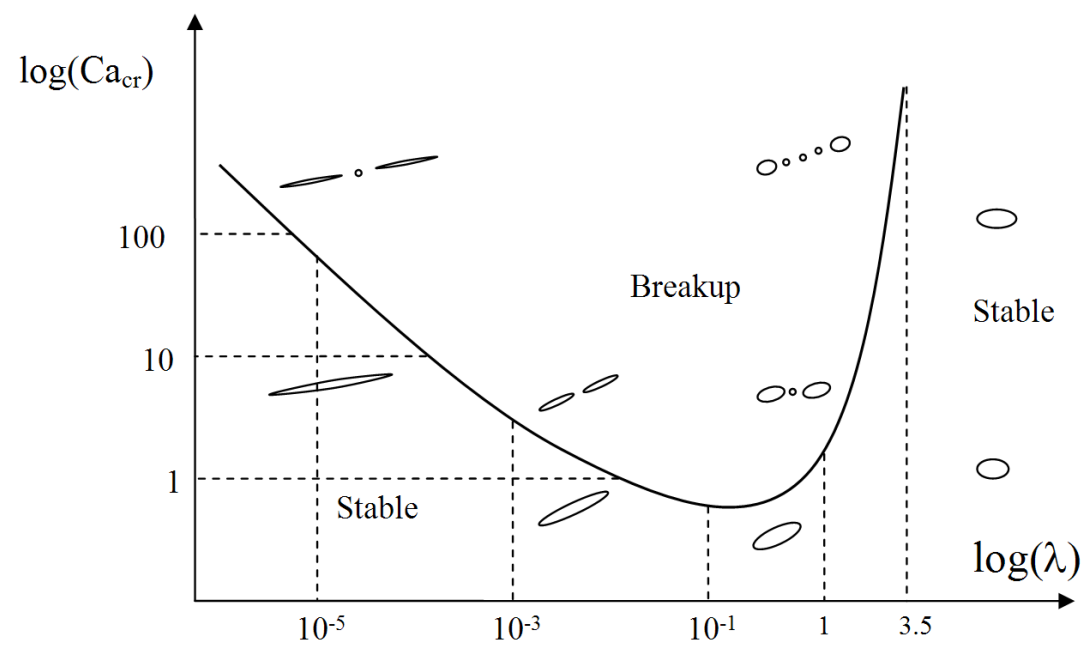

Figure 10: Critical value of the capillary number for breakup in shear flow. Also shown are the corresponding droplet shapes before and after breakup

experiments [30, 32]. Other theoretical approaches, as well as numerical methods have been proposed and are well documented in the review papers of Rallison [33] and Stone [34].

\section{RHEOLOGY OF VESICLE SUSPENSION AND COMPARISON TO DROPLET EMULSION}

Once the precise expression of the velocity field is obtained together with the vesicle shape evolution equation, the rheology of a dilute suspension can be analyzed. For that purpose, we average[35, 36] the stress over the total volume: $\left\langle\sigma_{i j}\right\rangle=\frac{1}{V} \int_{V} \sigma_{i j} d V$, which can be rewritten as

$$
\begin{aligned}
& \left\langle\sigma_{i j}\right\rangle=-\langle p\rangle \delta_{i j}+\eta_{0}\left\langle\frac{\partial v_{i}}{\partial x_{j}}+\frac{\partial v_{j}}{\partial x_{i}}\right\rangle \\
& +\frac{1}{V} \int_{V}\left[\sigma_{i j}-\eta_{0}\left(\frac{\partial v_{i}}{\partial x_{j}}+\frac{\partial v_{j}}{\partial x_{i}}\right)+p \delta_{i j}\right] d V .
\end{aligned}
$$

The last term represents the vesicles contribution, since it vanishes in their absence. Because of the stress jump at the membrane, we transform the volume integral into a surface one (lying on the external liquid side):

$$
\left\langle\sigma_{i j}\right\rangle=\eta_{0}\left\langle\frac{\partial v_{i}}{\partial x_{j}}+\frac{\partial v_{j}}{\partial x_{i}}\right\rangle
$$




$$
+\frac{1}{V} \int_{A}\left\{\sigma_{i k} x_{j} n_{k}-\eta_{0}\left(v_{i} n_{j}+v_{j} n_{i}\right)\right\} d A
$$

The second part describes the vesicle contribution. We have omitted $\langle p\rangle$ since it gives no contribution under pure shear. Using the hydrodynamical solution given in section $\mathrm{C}$, and plug into the above integral we find the following relation

$$
\left\langle\sigma_{i j}\right\rangle=\sigma_{i j}^{0}-3 \eta \phi T_{i j}
$$

where $\phi$ is the volume fraction of the suspension (the volume occupied by the vesicles over the total volume), and $\sigma_{i j}^{0}=2 \eta e_{i j}$ is the stress in the vesicle-free fluid. Actually relation (35) was obtained for droplets[26] and vesicles[38].

Using the relations between $T_{i j}$ and $F_{i j}$ from the use of the results of section C, together with Eq.(20), we can express fully $T_{i j}$ as a function of $F_{i j}$. Then using (35) leads to the following expression of the stress field (where now we omit the average symbol)

$$
\frac{\sigma_{i j}}{2 \eta_{0}}=e_{i j}+\phi\left(\frac{5}{2}-2 h \sqrt{\frac{15}{2 \pi}}\right) e_{i j}+\phi h \sqrt{\frac{15 \pi}{2}} \frac{96 F_{12} F_{i j}}{5 \Delta}
$$

The analogous expression in the case of droplets is given by[26]:

$$
\frac{\sigma_{i j}}{2 \eta_{0}}=e_{i j}+\frac{\phi}{2}\left[\frac{10(\lambda-1)}{2 \lambda+3} e_{i j}+\frac{24}{2 \lambda+3} F_{i j}\right]
$$

Here again the equation for vesicles is nonlinear in the deformation, while it is linear for drops. The source of the nonlinearity is again the membrane incompressibility.

We determine now the rheological properties, and set up a link between microscopic dynamics and rheology for vesicles. Of particular interest are the effective viscosity and the normal stress differences. In the tank-treading regime Eq.(20) can be solved analytically. We obtain

$$
F_{12}=\frac{1}{12 h} \sqrt{\frac{15 \Delta\left(4 h^{2}-\Delta\right)}{32 \pi}}
$$

This is also the same information as Eq.(26). Making use of Eq.(36), we find for the effective viscosity

$$
\eta_{\text {eff }}=\eta\left[1+\frac{5}{2} \phi-\phi \frac{23 \lambda+32}{16 \pi} \Delta\right]
$$

which extends the famous Einstein[37] result for particle suspensions to the case of vesicles. This result was originally derived in [9]. An inspection of this relation shows that $\eta_{\text {eff }}$ decreases upon decreasing $h$ (or equivalently it decreases with increasing viscosity ratio 
$\lambda$ ). The same holds for a fixed $h$ but increasing $\Delta$. This result can be understood from the expression of $\psi_{0}$. Indeed, the orientation angle decreases upon a decrease of $h$ (which corresponds to an increase of $\lambda$ ) or an increase of $\Delta$. This entails that the vesicle aligns more and more along the flow, resulting in a reduction of the viscosity. For $4 h^{2}=\Delta, \psi_{0} \rightarrow 0$, and the effective viscosity at the $t b$ threshold reads $\eta_{\text {eff }}=\eta[1+5 \phi / 2-\phi \sqrt{15 \Delta / 2 \pi}]$. For $\Delta=1$ (corresponding to $8 \%$ of relative excess area), we find $\eta_{\text {eff }} \simeq \eta(1+\phi)$, which is a significant shift with respect to the Einstein result. Even for $\Delta=0.2$, (less than $2 \%$ of relative excess area), we have $\eta_{\text {eff }} \simeq \eta(1+1.8 \phi)$. This is still a significant alteration of the Einstein result.

It is worth of mention that for $\Delta=0$ (the spherical limit), we obtain $\eta_{\text {eff }}=1+5 \phi / 2$, irrespective of the value of the viscosity ratio. This is a peculiar property of the presence of a membrane (even though it is fluid). Indeed due to the (fluid) membrane integrity the enclosed fluid executes a solid-like rotation, so that it behaves as a rigid body, explaining the above result. This property does not hold, for example, for a spherical droplet, as dissipation occurs within the enclosed fluid.

For drop emulsion, the effective viscosity is given by[22, 26]

$$
\eta_{\text {eff }}^{d}=1+\frac{1+5 \lambda / 2}{1+\lambda} \phi
$$

The functional dependence of the viscosity of droplet emulsions is quite different from that of a vesicle suspension. The first noticeable difference is that for droplets $\eta_{\text {eff }}^{d}$ increases with $\lambda$, while for vesicles $\eta_{\text {eff }}$ decreases with $\lambda$. This is true as a long the tank-treading regime is concerned.

The second important point is that while for large enough $\lambda, \eta_{\text {eff }}^{d}$ tends to $1+5 / 2 \phi$, this is not the case for vesicles (expect if $\Delta=0$ ). Indeed, as seen below, due to the $t b$ bifurcation, the vesicle suspension may exceed, even for quite small $\Delta$, the Einstein viscosity. The above expression for $\eta_{\text {eff }}$ is valid in the tank-treading regime. In the $t b$ and $v b$ regimes a time-dependent study is needed.

Let us now turn to the most general case of time-dependent dynamics. From the above rheological equations, the full expression of the viscosity is given by

$$
\eta_{e f f}=\eta\left[1+\frac{5}{2} \phi\left(1-\frac{4}{5} \sqrt{\frac{15}{2 \pi}} h\right)+\frac{\phi}{\Delta} h \sqrt{\frac{470}{\pi}} R^{2} \sin ^{2}(2 \psi)\right]
$$

In order to compute the normal stress differences $N_{1}=\sigma_{11}-\sigma_{22}$ and $N_{2}=\sigma_{22}-\sigma_{33}$ the 
determination of the diagonal elements of $F_{i j}$ is required. We obtain from (20)

$$
F_{11}=-F_{22}=\frac{\Delta}{192 \pi}(23 \lambda+32), \quad F_{33}=0
$$

so that (here we switch to physical variables)

$$
N_{1}=-2 N_{2}=\eta \dot{\gamma} \phi \sqrt{\frac{15 \Delta\left(4 h^{2}-\Delta\right)}{2 \pi h^{2}}}
$$

Here also it is seen that $N_{1}$ decreases with decreasing $h$ (or equivalently increasing $\lambda$ ).

What happens to the rheological properties when the $t b$ boundary in parameter space is crossed? While the tank-treading motion can be analyzed analytically down to the $t b$ bifurcation, in the $t b$ regime it has not been possible to obtain analytical expressions (due to the coupling between the shape of the vesicle, and the orientation angle). Thus Eq.(20) has been solved numerically. For definiteness we fix $\Delta=1$, and calculate $\eta_{\text {eff }}, N_{1}$ and $N_{2}$ for the three regimes: tank-treading, $t b$ and $v b$. In the last two regimes $\eta_{\text {eff }}$ and $N_{1}, N_{2}$ are nonlinear oscillating functions of time. We are interested here in the time average over one period. The results are reported on Fig. 10. While the $t b$ threshold is approached $\eta_{\text {eff }}$ undergoes a decline. In the $t b$ regime $\eta_{\text {eff }}$ exhibits a sudden increase (Fig11). $\eta_{\text {eff }}$ is found to exhibit a cusp singularity, $\left(\eta_{\text {eff }}-\eta_{0}\right) / \eta_{0} \phi \sim\left|\lambda-\lambda_{c}\right|$ at the critical viscosity ratio $\lambda_{c}$ at which $t b$ takes place. This singularity is believed to be a general feature, beyond the small deformation theory. It reflects the behavior of the fold catastrophe associated with the $t b$ bifurcation. Note that the VB mode shows a smaller viscosity than the $t b$ one. The $t b$ regime possesses a higher viscosity than the tank-treading one at the same distance from the bifurcation point. Several remarks are in order. The sudden increase of $\eta_{\text {eff }}$ in the $t b$ regime can be traced back to the fact that over a period the vesicles scan a larger cross section against the flow than in the tank-treading regime, and in the VB one. As one moves far into the $t b$ regime the period of rotation becomes smaller and smaller as compared to $\dot{\gamma}^{-1}$, so that on the time scale of the imposed flow $\dot{\gamma}^{-1}$, the flow capability is reduced further and further. The same reasoning holds for the VB mode.

It must be noted that real suspensions are polydisperse and it is likely that the cusp is smeared out due to this fact. Furthermore, if one includes the higher order contribution in the deformation then the results may be modified. For example, if one plots the effective viscosity at finite $\dot{\gamma}$ (and not taking the limit $\dot{\gamma} \rightarrow 0$ ), then $\eta_{\text {eff }}$ exhibits a smooth minimum 
at finite $C_{a}$. At small enough $C_{a}$, which corresponds to taking the limit $\dot{\gamma} \rightarrow 0$, the cusp survives. In that case the $t b$ transition is not preceded by the VB mode (see Fig.7).

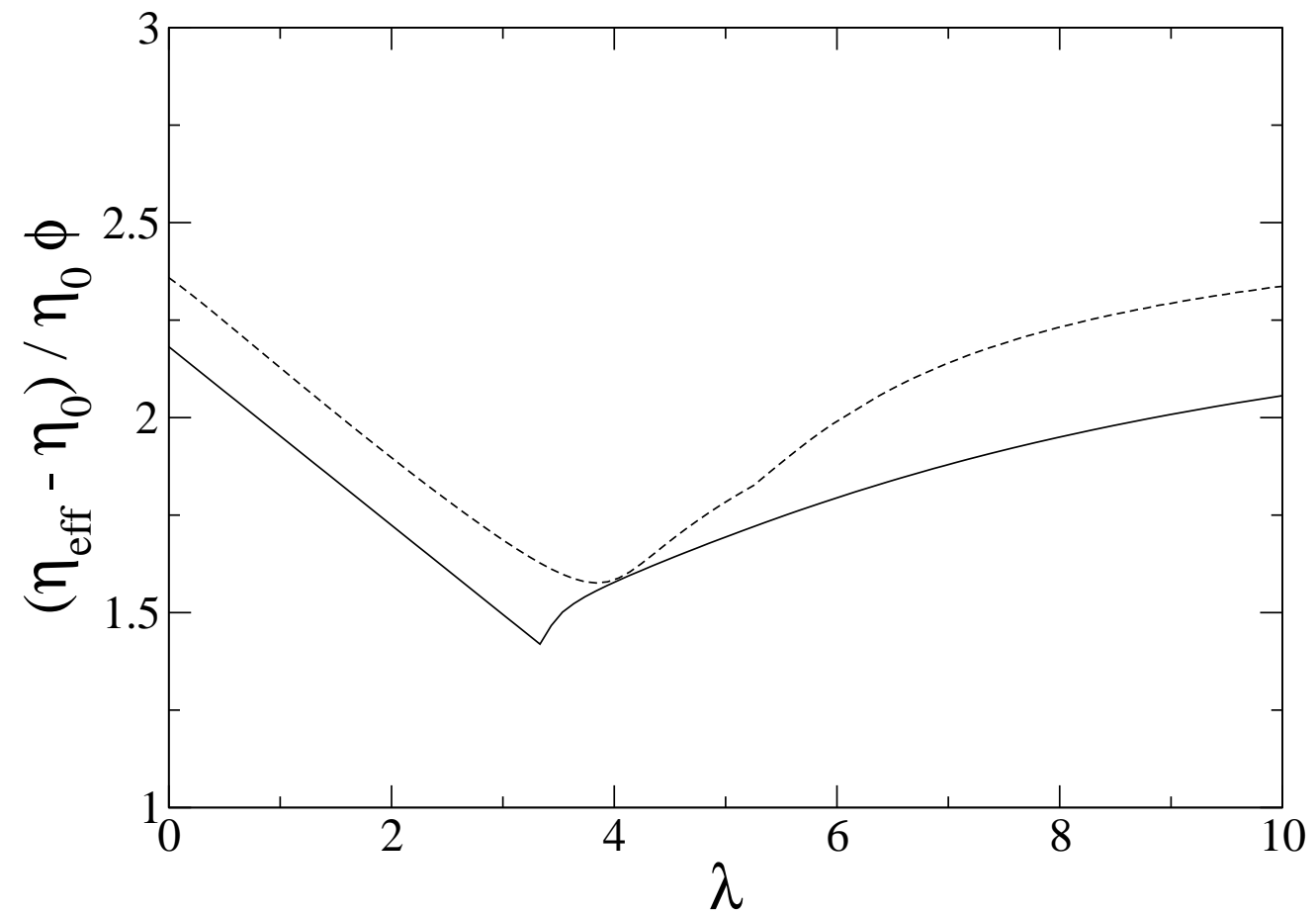

Figure 11: The reduced effective viscosity as a function of $\lambda$ for $C a=1$ and $\Delta=0.5$. The solid line is due to the leading-order theory presented here and shows a cusp singularity. The dashed line is according to the next-to-leading-order theory. Here the cusp singularity is smeared out unless $C a \ll 1$.

Another important feature is obtained from the analysis of $N_{1}$ and $N_{2}$ (averaged over a period). Both $N_{1}$ and $N_{2}$ undergo a collapse at the $t b$ threshold[38]. In the $t b$ and $v b$ regimes $N_{1}$ and $N_{2}$ remain very close to a zero value. From the tank-treading side they exhibit a square root singularity, which is directly connected with the behavior of the orientation angle. Normal stress differences are usually attributed to elongation along the flow of the suspended entities. Here, in the $t b$ regime, on the average, there is no preferred orientation of the vesicle, so that the fluid behaves from this perspective as does a Newtonian one. The higher order theory[12] shows that the picture remains the same for $C_{a}$ small enough. At large enough $C_{a}$ where the $v b$ mode takes place the square root singularity is smeared out[12]. We analyze here a new effect in that how $N_{1,2}$ behaves at fixed $\lambda$ by varying $C_{a}$. The result is reported on Fig.13. 


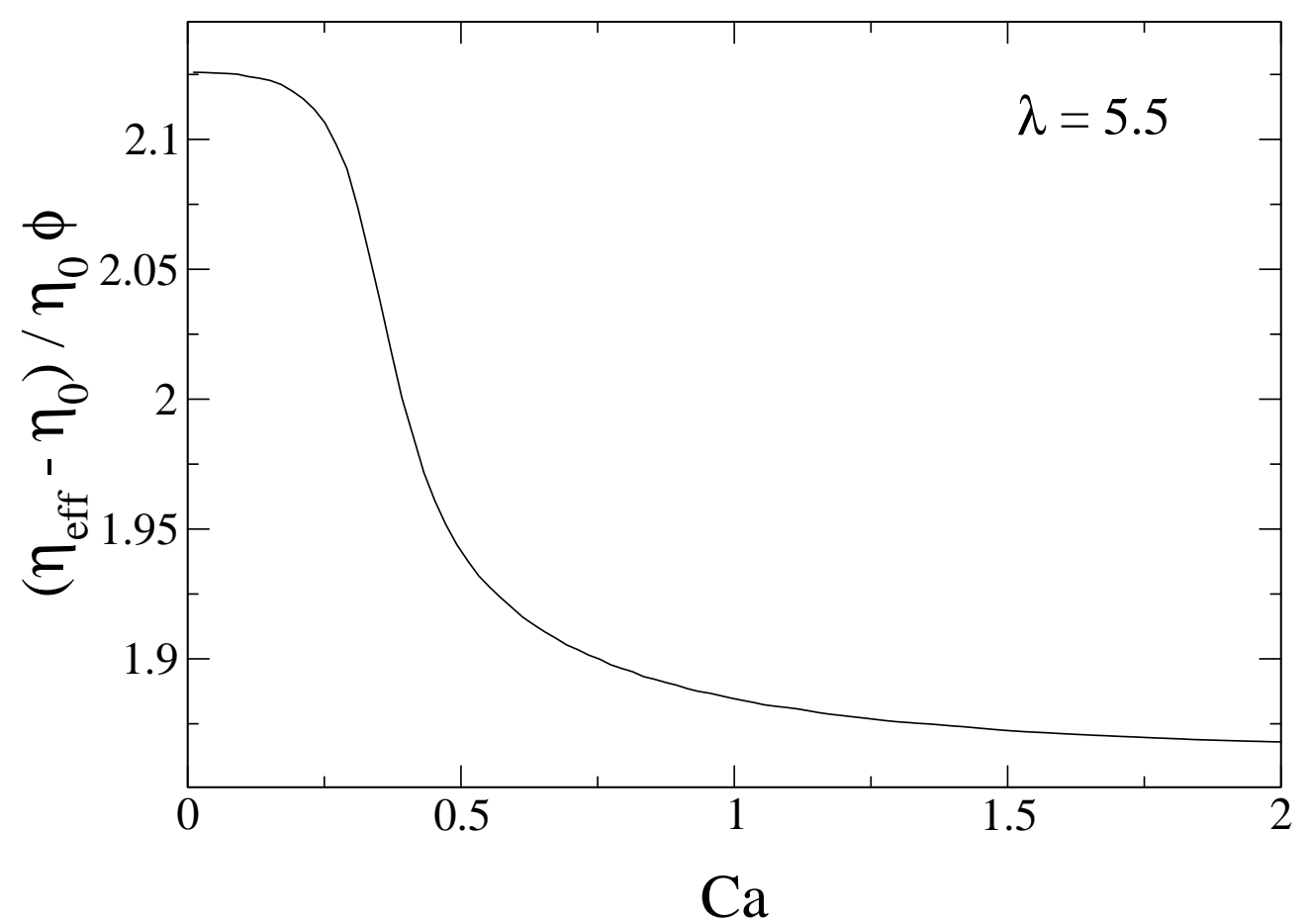

Figure 12: The reduced effective viscosity as a function of $C a$ for $\lambda=5.5$ and $\Delta=0.5$ according to the next-to-leading order theory. Parameters have been chosen such that the $t u-v b$ line is crossed in the phase diagram (see Fig. 7).

It is noteworthy that the normal stress differences $N_{1}$ and $N_{2}$ obtained for vesicle suspensions are linear in the shear rate $\dot{\gamma}$ and not quadratic as is the case for droplet emulsions [39]. This is traced back to the fact that the shape-dependent Lagrange multiplier $\zeta$ enters the shape evolution equation (20).

\section{CONCLUSIONS}

The first purpose of this paper was to analyze the similarity and dissimilarity between vesicle and drop dynamics. It has been shown that vesicles exhibit three distinct motions, while drops (as long as they maintain their integrity) show only one type of motion, namely steady orientation in the shear flow. We have given several physical explanations of the basic differences. The membrane incompressibility leads to nonlinearity in the evolution equation, and these nonlinearities trigger bifurcations and coexistence of modes ( $t b$ and $v b$ ). We have then derived a rheological law in terms of an average stress tensor. We have found that the effective viscosity behaves differently than the drop one. In addition due to the dynamical 


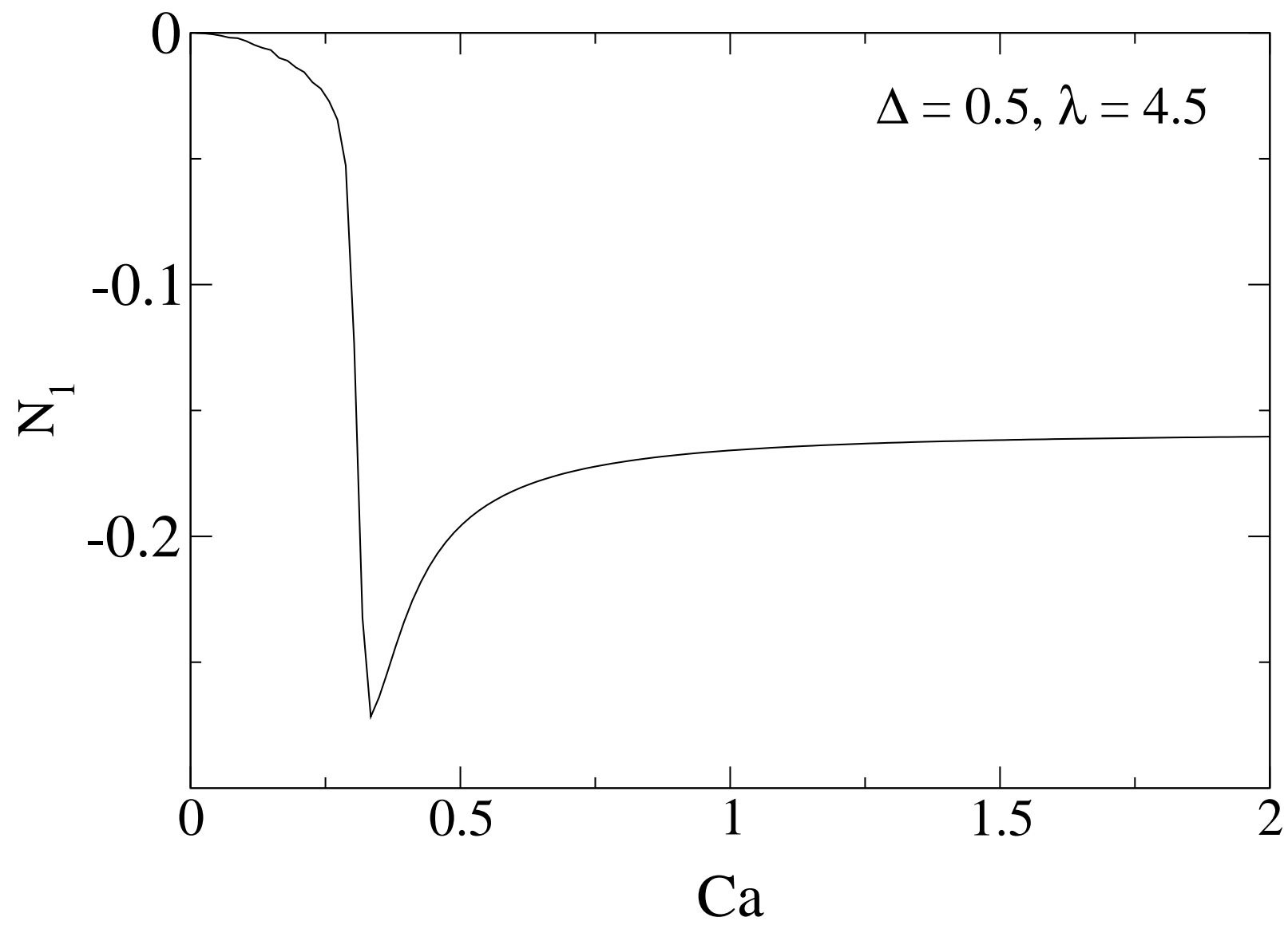

Figure 13: The first normal stress difference $N_{1}$ as a function of $h$ for the various three regimes: Tank-treading, Tumbling and Vacillating-Breathing $(v b)$.

regimes $(t b$ and $v b)$ the viscosity shows a cusp singularity at the bifurcation point, whereas the normal stress differences vanish as a square root when one goes from tank-treading to $t b$ and $v b$.

In the literature capsules have been studied theoretically in the small deformation limit[40]. Due to the fact that the authors start from a sphere, the shear flow may deform it only if one imposes a stretching of the area. This is why, the obtained evolution equation together with the rheological equation are linear, and therefore they do not exhibit the type of dynamics and bifurcation as do vesicles. Our trick was to assume a deviation from the sphere, and then to close the problem by saying that the available excess area must comply with the shape function. This leads to a drastic difference with capsule theories.

Finally it is an important task for future investigation to combine our trick of capturing nonlinearities and elastic effects, such as shear modes, with the aim to apply our theory to capsules and red blood cells. This should constitute a first step towards understanding 
blood rheology from microscopic considerations.

\section{ACKNOWLEDGEMENTS}

C. M. acknowledges financial supports from CNES (Centre National d'Etudes Spatiales), and from CNRS ("ACI de la cellule et du myocarde"). C. V. and G. D. acknowledge financial support from the European Commission Marie Curie Research Training Network MRTN-CT-2004-503661. 
[1] Helfrich W, Blocked lipid exchange in bilayers and its possible influence on the shape of vesicles. Z. Naturforsch. 29c (1974) 510-515.

[2] U. Seifert, Configurations of fluid membranes and vesicles, Adv. Phys. 46 (1997), 13-137.

[3] U. Seifert, K. Berndl, and R. Lipowsky, Shape transformations of vesicles: Phase diagram for spontaneous-curvature and bilayer-coupling models, Phys. Rev. A 44 (1991) 1182-1202.

[4] M. Kraus, W. Wintz, U. Seifert, and R. Lipowsky, Fluid vesicles in shear flow, Phys. Rev. Lett. 77 (1996) 3685-3688.

[5] Isabelle Durand, Petra Jönson, Chaouqi Misbah, Alexander Valance, and Klaus Kassner, Adhesion-induced vesicle propulsion, Phys. Rev. E 56 (1997) R3776-R3779; I. Cantat and C. Misbah, Lift force and dynamical unbinding of adhering vesicles under shear flow, Phys. Rev. Lett. 83 (1999) 880-883.

[6] U. Seifert, Hydrodynamic lift of bound vesicles, Phys. Rev. Lett. 83 (1999) 876-879; S. Sukumaran and U. Seifert, Influence of shear flow on vesicles near a wall: a numerical study, Phys. Rev. E 64 (2001) 011916.

[7] T. Biben, C. Misbah, Tumbling of vesicles under shear flow, Phys. Rev. E 67 (2003) 031908; J. Beaucourt, F. Rioual, T. Sèon, T. Biben, and C. Misbah, Steady to unsteady dynamics of a vesicle in a flow, Phys. Rev. E 69 (2004) 011906.

[8] H. Noguchi and G. Gompper, Fluid vesicles with viscous membranes in shear flow, Phys. Rev. Lett. 93 (2004) 258102.

[9] C. Misbah, Vacillating breathing and tumbling of vesicles under shear flow, Phys. Rev. Lett. 96 (2006) 028104.

[10] V. V. Lebedev, K. S. Turitsyn, and S. S. Vergeles, arXiv:cond-mat/0702650v1.

[11] H. Noguchi and G. Gompper, Phys. Rev. Lett. 98, 128103 (2007).

[12] G. Danker, T. Biben, T. Podgorski, C. Verdier and C. Misbah, arXiv:cond-mat/0703698v1.

[13] U. Seifert, Fluid membranes in hydrodynamic flow fields: formalism and an application to fluctuating quasispherical vesicles in shear flow, Eur. Phys. J. B. 8 (1999) 405-415.

[14] H. Lamb, Hydrodynamics, 6th edition, Cambridge Univ. Press, 1932.

[15] R.G. Cox, The deformation of a drop in a general time-dependent fluid flow, J. Fluid Mech. 37(3) (1969) 601-623. 
[16] K. de Haas, C. Bloom, D. van den Ende, M. Duits, J. Mellema, Deformation of giant lipid bilayer vesicles in shear flow, Phys. Rev. E 56, (1997) 7132-7137; B. Lorz, R. Simson, J. Nardi, E. Sackmann, Weakly adhering vesicles in shear flow: Tanktreading and anomalous lift force, Europhys. Lett. 51 (2000) 468-474.

[17] M. Abkarian, C. Lartigue, A. Viallat, Tanktreading and unbinding of deformable vesicles in shear flow: Determination of the lift force, Phys. Rev. Lett. 88 (2002) 068103.

[18] V. Vitkova, M. Mader, T. Podgorski, Deformation of vesicles flowing through capillaries, Europhys. Lett. 68 (2004) 398-404; M. Mader, V. Vitkova, M. Abkarian, A. Viallat, T. Podgorski, Dynamics of viscous vesicles in shear flow, Eur. Phys. J. E. 19 (2006) 389-397.

[19] V. Kanstler, V. Steinberg, Orientation and Dynamics of a Vesicle in Tank-Treading Motion in Shear Flow, Phys. Rev. Lett. 95 (2005) 258101; ibid, Transition to Tumbling and Two Regimes of Tumbling Motion of a Vesicle in Shear Flow, Phys. Rev. Lett.96 (2006) 036001.

[20] S. R. Keller and R. Skallak, Motion of a tank-treading ellipsoidal particle in a shear-flow, J. Fluid Mech. 120 (1982) 27-47.

[21] G.I. Taylor, The viscosity of a fluid containing small drops of another fluid, Proc. Royal Soc. A138 (1932) 41-48.

[22] G.I. Taylor, The formation of emulsions in definable fields of flow, Proc. Royal Soc. A146 (1934) 501-523.

[23] S. Torza, R.G. Cox, S.G. Mason, Particle motions in sheared suspensions. XXVII. Transient and steady deformation and burst of liquid drops, J. Colloid Int. Sci. 38 (1972) 395-411.

[24] J.D. Buckmaster, J.E. Flaherty, The bursting of two-dimensional drops in slow viscous flow, J. Fluid Mech. 60(4) (1973) 625-639.

[25] F.D. Rumscheidt, S.G. Mason, Particle motions in sheared suspensions. XII. deformation and burst of fluid drops in shear and hyperbolic flow, J. Colloid Sci. 16 (1961) 238-261.

[26] N.A. Frankel and A. Acrivos, The constitutive equation for a dilute emulsion, J. Fluid Mech. 44 (1970) 65-78.

[27] P.L. Maffettone, M. Minale, Equation of change for ellipsoidal drops in viscous flow, J. NonNewtonian Fluid Mech. 78 (1998) 227-241.

[28] P. Marmottant, T. Biben and S. Hilgenfeldt, Deformation and rupture of lipid vesicles in the shear flow generated by ultrasound-driven microbubbles, Preprint (2007).

[29] F. Brochard-Wyart, P.-G. de Gennes, and O. Sandre, Transient pores in stretched vesicles: 
role of leak-out, Physica A 278 (2000) 32-51.

[30] H.P. Grace, Dispersion phenomena in high viscosity immiscible fluid systems and application of static mixers as dispersion devices in such systems, Eng. Found., Res. Conf. Mixing, 3rd, Andover, N.H., 1971, republished in Chem. Eng. Commun. 14 (1982) 225-277.

[31] D. Barthès-Biesel, A. Acrivos, Deformation and burst of a liquid droplet freely suspended in a linear shear field, J. Fluid. Mech. 61(1) (1973) 1-21.

[32] B.J. Bentley, L.G. Leal, An experimental investigation of drop deformation and breakup in steady two-dimensional linear flows, J. Fluid Mech. 167 (1986) 241-283.

[33] J.M. Rallison, The deformation of small viscous drops and bubbles in shear flows, Ann. Rev. Fluid Mech. 16 (1984) 45-66.

[34] H.A. Stone, Dynamics of drop deformation and breakup in viscous fluids, Ann. Rev. Fluid Mech. 26 (1994) 65-102.

[35] L.D. Landau and E.M. Lifchitz, Fluid Mechanics, Pergamon Press, Oxford, 1993.

[36] G.K. Batchelor, The stress system in a suspension of force-free particles, J. Fluid. Mech. 41 (1970) 545-570.

[37] A. Einstein, Eine neue Bestimmung der Moleküldimensionen, Ann. Physik 19 (1906) 289-306;

A. Einstein, Berichtigung zu meiner Arbeit: Eine Neue Bestimmung der Moleküldimensionen, Ann. Physik 34 (1911) 591-592.

[38] G. Danker and C. Misbah, Rheology of a dilute suspension of vesicles, Phys. Rev. Lett. 98 (2007) 088104-1-088104-4.

[39] W. R. Schowalter, C. E. Chaffey, and H. Brenner, Rheological Behavior of a Dilute Emulsion, J. Colloid Interface Sci. 26 (1968) 152-160.

[40] D. Barthès-Biesel, J.M. Rallison, The time-dependent deformation of a capsule freely suspended in a linear shear flow, J. Fluid. Mech. 113 (1981) 251-267; A. Drochon, Rheology of dilute suspensions of red blood cells: experimental and theoretical approaches, Eur. Phys. J. AP 22 (2003) 155-162.

[41] Other candidates like $|H|$ could be considered. This singular behavior has no physical support.

[42] Note that the phase diagram is more complex than that shown on Fig.2. Indeed, there is at least a second new parameter which is the spontaneous curvature $H_{0}$ introduced in the model as $\kappa / 2 \int\left(H-H_{0}\right)^{2} d A$, and which states that the natural curvature for an open membrane would not be $H=0$ but $H=H_{0} \neq 0$, that is to say the membrane has a spontaneous curvature. 
Other models are also introduced in the literature; see [2])

[43] It must be noted that this is true for a clean drop. If there is an adsorbed film on the surface of the drop i.e. in the presence of surfactants [34], any motion can result in the appearance of additional forces (Marangoni) which have to be taken into account in the hydrodynamical boundary conditions. Further, proper convective-diffusion equations for surfactant transport need to be written. In this case, the dynamics of the drop may approach that of a vesicle. 